\begin{tabular}{|c|l|}
\hline Title & Periodic Model Predictive Control of Multi-Hop Control Networks \\
\hline Author(s) & Satoh, Dai; Kobay ashi, Koichi; Y amashita, Y uh \\
\hline Citation & $\begin{array}{l}\text { IEICE transactions on fundamentals of electronics communications and computer sciences, E100A (2), 406-413 } \\
\text { https://doi.org/L0.1587 transfun.E100.A.406 }\end{array}$ \\
\hline Issue Date & 2017-02 \\
\hline Doc URL & http://hdl.handle.net/2115/65210 \\
\hline Rights & copyright@2017 IEICE \\
\hline Type & article \\
\hline File Information & Periodic model predictive control of multi-hop control networks.pdf \\
\hline
\end{tabular}

Instructions for use 


\title{
Periodic Model Predictive Control of Multi-Hop Control Networks
}

\author{
Dai SATOH $^{\dagger}$, Nonmember, Koichi KOBAYASHI $^{\dagger a}$, and Yuh YAMASHITA $^{\dagger}$, Members
}

\begin{abstract}
SUMMARY In this paper, a new method of model predictive control (MPC) for a multi-hop control network (MHCN) is proposed. An MHCN is a control system in which plants and controllers are connected through a multi-hop wireless network. In the proposed method, (i) control inputs and (ii) paths used in transmission of control inputs are computed with constant period by solving the finite-time optimal control problem. First, a mathematical model for expressing an MHCN is proposed. This model is given by a switched linear system, and is compatible with MPC. Next, the finite-time optimal control problem using this model is formulated, and is reduced to a mixed integer quadratic programming problem. Finally, a numerical example is presented to show the effectiveness of the proposed method.

key words: multi-hop control networks, model predictive control, switched linear systems
\end{abstract}

\section{Introduction}

In recent years, there have been a lot of studies on networked control systems (NCSs) and cyber-physical systems (CPSs) [5], [13]. An NCS is a control system in which components such as plants and controllers are connected through a communication network. A CPS is a control system consisting of cyber components such as cloud servers and physical components such as mechanical systems. As one of the research topics of NCSs and CPSs, control of multiple plants over communication networks is important (see, e.g., [1]). In many applications, wireless networks are used. In particular, multi-hop wireless networks have attracted much attention (see, e.g., [8], [11], [18], [19]). Multi-hop wireless networks are generally composed of a large number of wireless nodes deployed randomly in a two (or three) dimensional space. In such networks, communications between nodes are achieved through multi-hop paths. Furthermore, a control system over a multi-hop wireless network is called a multi-hop control network (MHCN) [4]. In [4], its formal model has been proposed, and several properties have been analyzed. In [7], [10], fault tolerant control has been studied. In [15], [20], co-scheduling of communication and control has been studied. To the best of our knowledge, model predictive control (MPC) of MHCNs has not been studied so far. MPC is a control method that the control input is generated by solving the finite-time optimal control problem at

\footnotetext{
Manuscript received April 15, 2016.

Manuscript revised August 15, 2016.

$\dagger$ The authors are with the Graduate School of Information Science and Technology, Hokkaido University, Sapporo-shi, 060-0814 Japan.

a)E-mail: k-kobaya@ssi.ist.hokudai.ac.jp DOI: 10.1587/transfun.E100.A.406
}

each time (see, e.g., [9]). MPC has several benefits. One of the benefits is to obtain an optimal control input satisfying state/input constraints. In [21], a prediction control method over multi-hop networks has been proposed. In this method, prediction is utilized for delay compensation, but optimal control and state/input constraints are not considered. In [14], the optimal control problem has been studied, but MPC has not been considered.

In this paper, a new method of MPC for MHCNs is proposed. In the proposed method, we assume that the control input is recomputed with constant period, and the number of controllers is one. The length of period is given based on the length of paths from controllers to plants. Furthermore, the candidates of paths from controllers to plants are enumerated in advance. Hence, unnecessary candidates of paths can be excluded. For a fixed path from controllers to plants, time sequence of the state is modeled by a linear system. Since a linear system is derived for each candidate of paths, a given MHCN can be modeled by a switched linear system. Also in [4], a switched linear system is used as a model of MHCNs. In the models in [4], [14], a directed graph appeared in an $\mathrm{MHCN}$ is modeled, but in the proposed method, time sequence of the state for a fixed path is modeled off-line. Hence, the proposed model is compatible with the finite-time optimal control problem and MPC.

Furthermore, the switched linear system can be transformed into a mixed logical dynamical (MLD) system [6]. Using the MLD system obtained, the finite-time optimal control problem is formulated. Since the MLD system is given as a form of a linear constrained system with binary variables and continuous variables, this control problem can be rewritten as a mixed integer quadratic programming (MIQP) problem, which can be solved by a suitable free/commercial solver.

The proposed method provides us a basic result for MPC of MHCNs.

This paper is organized as follows. In Sect. 2, the notion of MHCNs is defined according to [4], [14]. In Sect. 3, the key idea of the proposed periodic MPC method is explained. In Sect. 4, a modeling method of MHCNs is proposed. In Sect. 5, the finite-time optimal control problem is formulated, and is reduced to an MIQP problem. In Sect. 6, a numerical example is presented. In Sect. 7, we conclude this paper.

Notation: Let $\mathcal{R}$ denote the set of real numbers. Let $\{0,1\}^{n}$ denote the set of $n$-dimensional vectors, which consists of elements 0 and 1 . Let $I_{n}$ and $0_{m \times n}$ denote the $n \times n$ identity matrix and $m \times n$ zero matrix, respectively. For sim- 


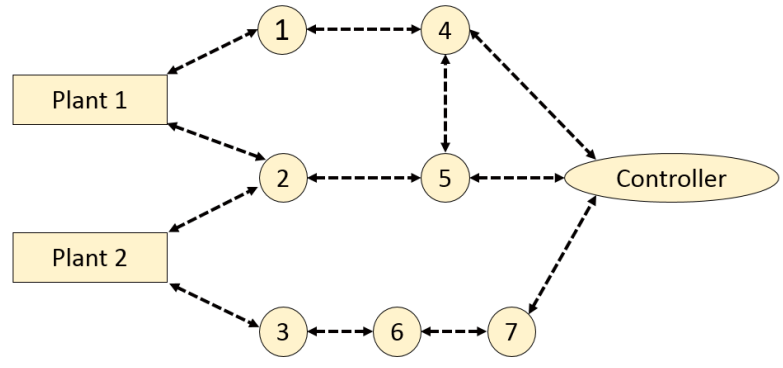

Fig. 1 Example of MHCNs.

plicity, we sometimes use the symbol 0 instead of $0_{m \times n}$, and the symbol $I$ instead of $I_{n}$. For the vector $v$, let $v^{\top}$ denote the transpose of $v$. For the finite set $A$, let $|A|$ denote the number of elements of $A$.

\section{Multi-Hop Control Networks}

In this section, a Multi-Hop Control Network (MHCN) is explained. An MHCN is illustrated by a directed graph such as Fig. 1. Nodes in MHCNs consist of three kinds of nodes, i.e., (i) nodes corresponding to plants, (ii) nodes corresponding to controllers, and (iii) nodes that communicate messages about sensing and actuating from plants (controllers) to controllers (plants). In the example of Fig. 1, there are two plants and one controller. In this paper, we assume that the number of controllers is given by 1 , and the dynamics of plants are given by a discrete-time linear system. Messages about the control input are sent from the controller to each plant according to the directed graph in MHCNs. In a similar way, messages about the measured signal is sent from each plant to the controller. In this paper, we suppose that a routing protocol is given by a kind of proactive routing protocols (see, e.g., [3]), and the set of available paths from a controller (plants) to plants (a controller) is given by the directed graph of the MHCN in advance. Such set can be frequently computed (see, e.g., [12], [16], [17]).

Here, an MHCN studied in this paper is formally defined as follows.

Definition 1: An MHCN is given by a tuple

$$
\mathcal{N}=\left(\mathcal{G}, \Sigma_{i}\right),
$$

where:

- $\mathcal{G}=(\mathcal{V}, \mathcal{E})$ is an undirected graph that expresses the radio connectivity of the network, where $\mathcal{V}$ is the set of vertices (nodes), and $\mathcal{E} \subseteq \mathcal{V} \times \mathcal{V}$ is the set of edges. The set $\mathcal{V}$ is decomposed into $\mathcal{V}=\mathcal{P} \cup \mathcal{C} \cup \mathcal{H}$, where

- $\mathcal{P}$ is the set of plants,

- $C$ is the set of controllers (assume $|C|=1$ ),

- $\mathcal{H}$ is the set of nodes that communicate messages.

For two nodes $i, j$, if $i$ is connected to $j$ (i.e., $j$ is connected to $i)$, then the message included in $i(j)$ may be sent to $j(i)$.
- $\Sigma_{i}, i=1,2, \ldots,|\mathcal{P}|$ express the dynamics of plants, that is,

$$
\Sigma_{i}: x_{i}(k+1)=A_{i} x_{i}(k)+B_{i} u_{i}(k)
$$

where $k \in\{0,1, \ldots\}$ is the discrete time, and $x_{i}(k) \in$ $\left[\underline{x}_{i}, \bar{x}_{i}\right] \subseteq \mathcal{R}^{n_{i}}$ and $u_{i}(k) \in\left[\underline{u}_{i}, \bar{u}_{i}\right] \subseteq \mathcal{R}^{m_{i}}$ are the state and the control input of the plant $i$, respectively $\left(\left[\underline{x}_{i}, \bar{x}_{i}\right]\right.$ and $\left[u_{i}, \bar{u}_{i}\right]$ are a given set). The matrices $A_{i} \in \mathcal{R}^{n_{i} \times n_{i}}$ and $B_{i} \in \mathcal{R}^{n_{i} \times m_{i}}$ are given in advance.

This definition is based on that in [4], [14]. In [4], the controller is given by a linear system. In [14], the controller is given by a kind of optimizer. In a similar way to [14], the controller studied in this paper is given by a kind of optimizer. However, in the method proposed in [14], only open-loop control has been considered. In the proposed method, closed-loop control based on model predictive control (MPC) [9] is considered.

\section{Periodic Model Predictive Control}

In this section, periodic MPC for MHCNs, which is the key idea of this paper, is explained.

Conventional control of MHCNs is conducted according to the following procedure.

\section{Conventional procedure of closed-loop control for MHCNs:}

Step 0: Preset the initial control input for each plant in advance.

Step 1: For each plant, compute

(i) control inputs,

(ii) paths that messages about control inputs are sent to plants.

Step 2: Send messages about the control input to each plant through the chosen path. After the message about the next control input reaches to the node corresponding to the plant, apply the next control input to the plant.

Step 3: Send messages about the measured state to the controller.

Step 4: Return to Step 1, if the controller receives messages about the measured state.

When MPC is implemented using the above conventional procedure, we consider the following technical problems.

(i) In Step 1, a method for finding a control input and a path.

(ii) In Step 3, a method for choosing a path from each plant to the controller.

(iii) In Step 4, the timing that the control input is recomputed (i.e., the timing that the procedure returns from Step 4 to Step 1) is not clear. In general, the controller receives messages from each plant at the different time.

In order to overcome (i), the optimal control problem using 
a mixed logical dynamical system has been formulated [14]. However, in [14], other issues have not been considered.

In order to overcome these issues, a periodic MPC method is proposed. To the best of our knowledge, MPC for MHCNs has not been studied so far. In the proposed method, the following assumptions are made for MHCNs.

Assumption 1: All nodes included in $\mathcal{H}$ can store only one message.

Assumption 2: Paths used in sending messages about the control input have no common nodes.

Assumption 3: When messages about the measured state are sent to the controller, paths used are the same as paths used in sending messages about the control input.

Assumption 4: A message sent from some node at time $k$ reaches to other adjacent node at time $k+1$.

Assumption 4 implies that communication delay from some node to other adjacent node is given by 1 . Let $L$ denote the maximum length of the path from the controller to plants. Then, the proposed procedure of periodic MPC is presented as follows.

\section{Procedure of periodic MPC for MHCNs:}

Step 0: Preset the initial control input for each plant in advance.

Step 1: For each plant, compute

(i) control inputs,

(ii) paths that messages about control inputs are sent to plants

by solving the finite-time optimal control problem. Set $t=0$.

Step 2: Send messages about the control input to each plant through the chosen path. After the message about the next control input reaches to the node corresponding to the plant, apply the next control input to the plant. The control input for each plant is updated until $t=L$.

Step 3: Send messages about the measured state to the controller, where paths used are the same as paths used in sending messages about the control input.

Step 4: Wait until time $t=2 L$. Then, the controller can receive messages about the measured state from each plant. Return to Step 1.

The outline of a modeling method and a finite-time optimal control problem is explained as follows.

Example 1: Consider the MHCN in Fig. 1. Based on this figure, we set $L=4$. The candidates of paths from Controller to Plant 1 are given by Fig. 2. The number of the candidates of paths is four. In a similar way, The candidates of paths from Controller to Plant 2 are given by Fig. 3. The number of the candidates of paths is three. Noting that Assumption 1 and Assumption 2 must be satisfied, from these figures we can obtain the following cases.

(i) Messages about the control input are not sent.

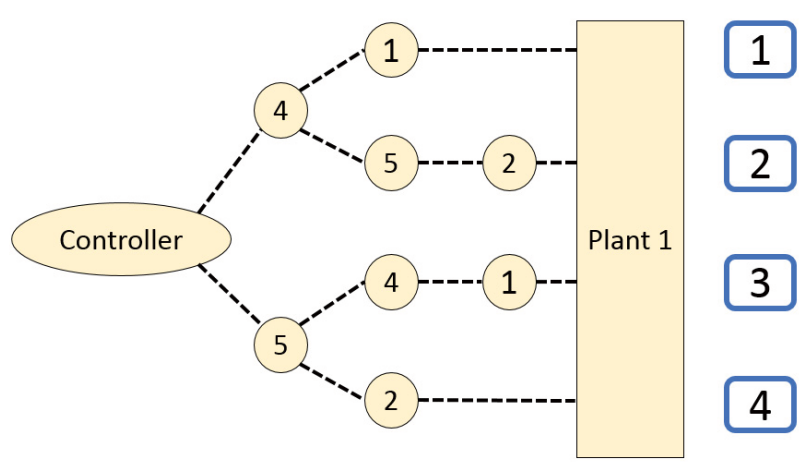

Fig. 2 The candidates of paths from Controller to Plant 1.

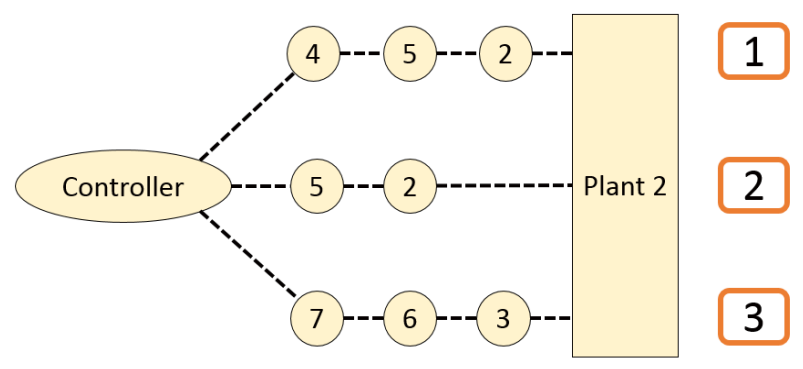

Fig. 3 The candidates of paths from Controller to Plant 2.

(ii) The message about the control input is sent to only Plant 1 through either Path 1 or Path 4.

(iii) The message about the control input is sent to only Plant 1 through either Path 2 or Path 3.

(iv) The message about the control input is sent to only Plant 2 through Path 2.

(v) The message about the control input is sent to only Plant 2 through either Path 1 or Path 3.

(vi) The message about the control input is sent to only Plant 1 through Path 1 , and the message about the control input is sent to only Plant 2 through Path 2.

(vii) Path 1 for Plant 1 and Path 3 for Plant 2 are chosen, or Path 4 for Plant 1 and Path 3 for Plant 2 are chosen.

(viii) Path 2 for Plant 1 and Path 3 for Plant 2 are chosen, or Path 3 for Plant 1 and Path 3 for Plant 2 are chosen.

For each case, we can obtain a constrained linear system. Hence, a given MHCN can be expressed by a switched linear system. The finite-time optimal control problem of Step 1 of the above procedure is to find a time sequence of the control input and a time sequence of the case minimizing a given cost function.

We remark here that all cases may not be enumerated. Since it is desirable to choose paths with a shorter length, a pair of paths with the longest length may not be a reasonable pair. Hence, such paths may be excluded from the candidates of paths. In enumeration of candidates of paths, the shortest path must be included in the set of candidates, but the longest path may not be included in the set of candidates. Considering the computation time for solving the finite-time 
optimal control problem, we must decide the set of candidates. Hereafter, an enumerated case is called a mode. In Sect. 4, we will explain the detail of a modeling method. In Sect. 5, we will explain the detail of the finite-time optimal control problem.

Remark 1: In this paper, we assume that the number of controllers is given by 1 . The proposed method can be extended to the case of multiple controllers in the following situation: (i) Assumption 1 is excluded, (ii) For each controller, plants are assigned, and there are no shared plants. When Assumption 1 is imposed, there is a possibility that message collision and loss occur.

Remark 2: Also in [4], a given MHCN is modeled by a switched linear system, but it is difficult to directly apply it to MPC. The proposed modeling method can be easily applied to MPC.

\section{Modeling Method}

An MHCN is modeled by using the following procedure.

\section{Procedure of modeling an MHCN:}

Step 1: Enumerate the candidates of paths from the controller to each plant.

Step 2: Enumerate the candidates of modes based on the obtained candidates.

Step 3: For each mode, derive a linear system in the time interval $[0,2 L]$.

Step 1 and Step 2 have been already explained in Example 1. In this section, Step 3 is explained using Example 1.

Example 2: Consider the MHCN in Figs. 1-3 again. Here, consider modeling a linear system for mode (vii) (i.e., Path 1 for Plant 1 and Path 3 for Plant 2 are chosen, or Path 4 for Plant 1 and Path 3 for Plant 2 are chosen). Then, noting that $2 L=8$, we can obtain

$$
\begin{aligned}
& {\left[\begin{array}{l}
x_{1}(1) \\
x_{2}(1)
\end{array}\right]=\left[\begin{array}{cc}
A_{1} & 0 \\
0 & A_{2}
\end{array}\right]\left[\begin{array}{l}
x_{1}(0) \\
x_{2}(0)
\end{array}\right]+\left[\begin{array}{cc}
B_{1} & 0 \\
0 & B_{2}
\end{array}\right]\left[\begin{array}{l}
u_{1}(-1) \\
u_{2}(-1)
\end{array}\right],} \\
& {\left[\begin{array}{l}
x_{1}(2) \\
x_{2}(2)
\end{array}\right]=\left[\begin{array}{cc}
A_{1} & 0 \\
0 & A_{2}
\end{array}\right]\left[\begin{array}{l}
x_{1}(1) \\
x_{2}(1)
\end{array}\right]+\left[\begin{array}{cc}
B_{1} & 0 \\
0 & B_{2}
\end{array}\right]\left[\begin{array}{l}
u_{1}(-1) \\
u_{2}(-1)
\end{array}\right],} \\
& {\left[\begin{array}{l}
x_{1}(3) \\
x_{2}(3)
\end{array}\right]=\left[\begin{array}{cc}
A_{1} & 0 \\
0 & A_{2}
\end{array}\right]\left[\begin{array}{l}
x_{1}(2) \\
x_{2}(2)
\end{array}\right]+\left[\begin{array}{cc}
B_{1} & 0 \\
0 & B_{2}
\end{array}\right]\left[\begin{array}{l}
u_{1}(-1) \\
u_{2}(-1)
\end{array}\right],} \\
& {\left[\begin{array}{l}
x_{1}(4) \\
x_{2}(4)
\end{array}\right]=\left[\begin{array}{cc}
A_{1} & 0 \\
0 & A_{2}
\end{array}\right]\left[\begin{array}{l}
x_{1}(3) \\
x_{2}(3)
\end{array}\right]+\left[\begin{array}{cc}
B_{1} & 0 \\
0 & B_{2}
\end{array}\right]\left[\begin{array}{l}
u_{1}(0) \\
u_{2}(-1)
\end{array}\right],} \\
& {\left[\begin{array}{l}
x_{1}(5) \\
x_{2}(5)
\end{array}\right]=\left[\begin{array}{cc}
A_{1} & 0 \\
0 & A_{2}
\end{array}\right]\left[\begin{array}{l}
x_{1}(4) \\
x_{2}(4)
\end{array}\right]+\left[\begin{array}{cc}
B_{1} & 0 \\
0 & B_{2}
\end{array}\right]\left[\begin{array}{l}
u_{1}(0) \\
u_{2}(0)
\end{array}\right],} \\
& {\left[\begin{array}{l}
x_{1}(6) \\
x_{2}(6)
\end{array}\right]=\left[\begin{array}{cc}
A_{1} & 0 \\
0 & A_{2}
\end{array}\right]\left[\begin{array}{l}
x_{1}(5) \\
x_{2}(5)
\end{array}\right]+\left[\begin{array}{cc}
B_{1} & 0 \\
0 & B_{2}
\end{array}\right]\left[\begin{array}{l}
u_{1}(0) \\
u_{2}(0)
\end{array}\right],} \\
& {\left[\begin{array}{l}
x_{1}(7) \\
x_{2}(7)
\end{array}\right]=\left[\begin{array}{cc}
A_{1} & 0 \\
0 & A_{2}
\end{array}\right]\left[\begin{array}{l}
x_{1}(6) \\
x_{2}(6)
\end{array}\right]+\left[\begin{array}{cc}
B_{1} & 0 \\
0 & B_{2}
\end{array}\right]\left[\begin{array}{l}
u_{1}(0) \\
u_{2}(0)
\end{array}\right],} \\
& {\left[\begin{array}{l}
x_{1}(8) \\
x_{2}(8)
\end{array}\right]=\left[\begin{array}{cc}
A_{1} & 0 \\
0 & A_{2}
\end{array}\right]\left[\begin{array}{l}
x_{1}(7) \\
x_{2}(7)
\end{array}\right]+\left[\begin{array}{cc}
B_{1} & 0 \\
0 & B_{2}
\end{array}\right]\left[\begin{array}{l}
u_{1}(0) \\
u_{2}(0)
\end{array}\right],}
\end{aligned}
$$

where $u_{1}(-1)$ and $u_{2}(-1)$ are the initial control input given in advance, and $u_{1}(0)$ and $u_{2}(0)$ are the control input, which is a decision variable in the finite-time optimal control problem. The control input for Plant 1 is updated at time 3 . The control input for Plant 2 is updated at time 4. From the above expressions, we can obtain the following linear system for mode (vii):

$$
x(1)=A x(0)+B_{7}^{0} u(0)+B_{7}^{-1} u(-1),
$$

where

$$
\begin{aligned}
& x(1)=\left[\begin{array}{c}
\hat{x}(1) \\
\hat{x}(2) \\
\hat{x}(3) \\
\hat{x}(4) \\
\hat{x}(5) \\
\hat{x}(6) \\
\hat{x}(7) \\
\hat{x}(8)
\end{array}\right], \quad x(0)=\left[\begin{array}{c}
\hat{x}(-7) \\
\hat{x}(-6) \\
\hat{x}(-5) \\
\hat{x}(-4) \\
\hat{x}(-3) \\
\hat{x}(-2) \\
\hat{x}(-1) \\
\hat{x}(0)
\end{array}\right], \quad \hat{x}(k)=\left[\begin{array}{l}
x_{1}(k) \\
x_{2}(k)
\end{array}\right], \\
& u(0)=\left[\begin{array}{l}
u_{1}(0) \\
u_{2}(0)
\end{array}\right], u(-1)=\left[\begin{array}{l}
u_{1}(-1) \\
u_{2}(-1)
\end{array}\right] \text {, } \\
& A=\left[\begin{array}{cccc}
0 & \cdots & 0 & \hat{A} \\
0 & \cdots & 0 & \hat{A}^{2} \\
\vdots & \vdots & \vdots & \vdots \\
0 & \cdots & 0 & \hat{A}^{8}
\end{array}\right], \quad \hat{A}=\left[\begin{array}{cc}
A_{1} & 0 \\
0 & A_{2}
\end{array}\right] \text {, }
\end{aligned}
$$

$$
B_{7}^{0}=\left[\begin{array}{c}
0 \\
0 \\
0 \\
\hat{A} B_{1}^{\prime} \\
\hat{B}+\hat{A} B_{1}^{\prime} \\
(I+\hat{A}) \hat{B}+\hat{A}^{2} B_{1}^{\prime} \\
\left(I+\hat{A}+\hat{A}^{2}\right) \hat{B}+\hat{A}^{3} B_{1}^{\prime} \\
\left(I+\hat{A}+\hat{A}^{2}+\hat{A}^{3}\right) \hat{B}+\hat{A}^{4} B_{1}^{\prime}
\end{array}\right],
$$$$
B_{1}^{\prime}=\left[\begin{array}{cc}
B_{1} & 0 \\
0 & 0
\end{array}\right], \quad \hat{B}=\left[\begin{array}{cc}
B_{1} & 0 \\
0 & B_{2}
\end{array}\right] \text {, }
$$

$$
B_{7}^{-1}=\left[\begin{array}{c}
\hat{B} \\
(I+\hat{A}) \hat{B} \\
\left(I+\hat{A}+\hat{A}^{2}\right) \hat{B} \\
B_{2}^{\prime}+\left(\hat{A}+\hat{A}^{2}+\hat{A}^{3}\right) \hat{B} \\
\hat{A} B_{2}^{\prime}+\left(\hat{A}^{2}+\hat{A}^{3}+\hat{A}^{4}\right) \hat{B} \\
\hat{A}^{2} B_{2}^{\prime}+\left(\hat{A}^{3}+\hat{A}^{4}+\hat{A}^{5}\right) \hat{B} \\
\hat{A}^{3} B_{2}^{\prime}+\left(\hat{A}^{4}+\hat{A}^{5}+\hat{A}^{6}\right) \hat{B} \\
\hat{A}^{4} B_{2}^{\prime}+\left(\hat{A}^{5}+\hat{A}^{6}+\hat{A}^{7}\right) \hat{B}
\end{array}\right],
$$

and

$$
B_{2}^{\prime}=\left[\begin{array}{cc}
0 & 0 \\
0 & B_{2}
\end{array}\right] \text {. }
$$

From this example, we see that the dynamics for each mode can be modeled by a linear system. Next, we derive a general form of a linear system for each mode. Let $l \in$ $\{0,1,2, \ldots$,$\} denote the label for cycles of communication.$ For each $l$, both the control input and the path are calculated. 
In general, the linear system for the mode $I$ can be obtained by

$$
x(l+1)=A x(l)+B_{I}^{0} u(l)+B_{I}^{-1} u(l-1),
$$

where

$$
\begin{aligned}
x(l) & =\left[\begin{array}{c}
\hat{x}(2 L l-2 L+1) \\
\hat{x}(2 L l-2 L+2) \\
\vdots \\
\hat{x}(2 L l)
\end{array}\right], \hat{x}(k)=\left[\begin{array}{c}
x_{1}(k) \\
x_{2}(k) \\
\vdots \\
x_{|\mathcal{P}|}(k)
\end{array}\right], \\
u(l) & =\left[\begin{array}{c}
u_{1}(l) \\
u_{2}(l) \\
\vdots \\
u_{|\mathcal{P}|}(l)
\end{array}\right], \\
A & =\left[\begin{array}{cccc}
0 & \cdots & 0 & \hat{A} \\
0 & \cdots & 0 & \hat{A}^{2} \\
\vdots & \vdots & \vdots & \vdots \\
0 & \cdots & 0 & \hat{A}^{2 L}
\end{array}\right], \\
\hat{A} & =\left[\begin{array}{cccc}
A_{1} & & & \\
& A_{2} & & \\
0 & & & \\
0 & & A_{|\mathcal{P}|}
\end{array}\right] .
\end{aligned}
$$

Since the matrices $B_{I}^{0}$ and $B_{I}^{-1}$ are different for each mode, these are omitted. The matrix $A$ in (1) does not depend on the mode. The mode $I$ in (1) may be different for each $l$. Hereafter, the mode $I$ in (1) is replaced with $I(l)$, and the set of modes is denoted by $\mathcal{M}=\{1,2, \ldots, M\}$. Thus, an MHCN can be modeled by the switched linear system with delay (1).

\section{Finite-Time Optimal Control Problem}

For the switched linear system with delay (1) expressing an $\mathrm{MHCN}$, consider the following finite-time optimal control problem.

Problem 1: For the switched linear system with delay (1) expressing an MHCN, suppose that the initial control input $u(-1)$, the initial state $x(0)$, and the prediction horizon $N$ are given. Then, find a control input sequence $u(0), u(1), \ldots, u(N-1)$ and a mode sequence $I(0), I(1), \ldots, I(N-1) \in \mathcal{M}$ minimizing the following cost function:

$$
J=\sum_{l=0}^{N-1}\left\{x^{\top}(l) Q x(l)+u^{\top}(l) R u(l)\right\},
$$

where $Q \geq 0$ and $R>0$ are a given weighting matrix.

This problem can be rewritten as a mixed integer quadratic programming (MIQP) problem. In order to explain this fact, we consider transforming (1) into a mixed logical dynamical (MLD) system [6].

First, define $\bar{x}(l):=\left[\begin{array}{ll}x^{\top}(l) & u^{\top}(l-1)\end{array}\right]^{\top}$. Then, (1) can be rewritten as

$$
\bar{x}(l+1)=\bar{A}_{I(l)} \bar{x}(l)+\bar{B}_{I(l)} u(l),
$$

where

$$
\bar{A}_{I(l)}=\left[\begin{array}{cc}
A & B_{I(l)}^{-1} \\
0 & 0
\end{array}\right], \quad \bar{B}_{I(l)}=\left[\begin{array}{c}
B_{I(l)}^{0} \\
0
\end{array}\right] .
$$

Next, a binary variable $\delta_{i}(l) \in\{0,1\}, i=1,2, \ldots, M$ is defined as follows:

$$
[I(l)=i] \leftrightarrow\left[\delta_{i}(l)=1\right], \quad i \in \mathcal{M} .
$$

Then, the equality constraint

$$
\delta_{1}(l)+\delta_{2}(l)+\cdots+\delta_{M}(l)=1, \quad l=1,2, \ldots, N-1
$$

must be imposed. Using a binary variable, (2) can be rewritten as

$$
\begin{aligned}
\bar{x}(l+1) & =\sum_{i=1}^{M} z_{i}(l), \\
z_{i}(l) & =\delta_{i}(l)\left\{\bar{A}_{i} \bar{x}(l)+\bar{B}_{i} u(l)\right\} .
\end{aligned}
$$

In addition, (5) is equivalent to the following linear inequalities

$$
\begin{aligned}
& g_{\min }^{i} \delta_{i}(l) \leq z_{i}(l) \leq g_{\max }^{i} \delta_{i}(l), \\
& \left(\bar{A}_{i} \bar{x}(l)+\bar{B}_{i} u(l)\right)-g_{\max }^{i}\left(1-\delta_{i}(l)\right) \\
& \quad \leq z_{i}(l) \leq\left(\bar{A}_{i} \bar{x}(l)+\bar{B}_{i} u(l)\right)-g_{\min }^{i}\left(1-\delta_{i}(l)\right) .
\end{aligned}
$$

where $g_{\min }^{i}$ and $g_{\max }^{i}$ are constant vectors such that for any $x_{i}(k) \in\left[\underline{x}_{i}, \bar{x}_{i}\right]$ and $u_{i}(k) \in\left[\underline{u}_{i}, \bar{u}_{i}\right] \subseteq \mathcal{R}^{m_{i}}$,

$$
g_{\min }^{i} \leq \bar{A}_{i} \bar{x}(l)+\bar{B}_{i} u(l) \leq g_{\max }^{i}
$$

is satisfied. Thus, the MLD system expressing (1) can be obtained as (3), (4), (6), and (7). We remark here that the MLD system obtained is linear with respect to $\bar{x}(l), z_{i}(l)$, and $\delta_{i}(l)$.

Using the MLD system obtained, Problem 1 can be rewritten as the following problem.

Problem 2: For the MLD system (3), (4), (6), and (7), suppose that the initial state $\bar{x}(0)$ and the prediction horizon $N$ are given. Then, find continuous decision variables $u(0), u(1), \ldots, u(N-1), z_{i}(0), z_{i}(1), \ldots, z_{i}(N-1)$ and binary decision variables $\delta_{i}(0), \delta_{i}(1), \ldots, \delta_{i}(N-1)$ minimizing the following cost function:

$$
J=\sum_{l=0}^{N-1}\left\{\bar{x}^{\top}(l)\left[\begin{array}{ll}
Q & 0 \\
0 & 0
\end{array}\right] \bar{x}(l)+u^{\top}(l) R u(l)\right\} .
$$

By a simple calculation, Problem 2 can be equivalently transformed into an MIQP problem, which can be solved by using a suitable free/commercial solver.

\section{Numerical Example}

As an example, consider the MHCN in Fig. 4, where the 


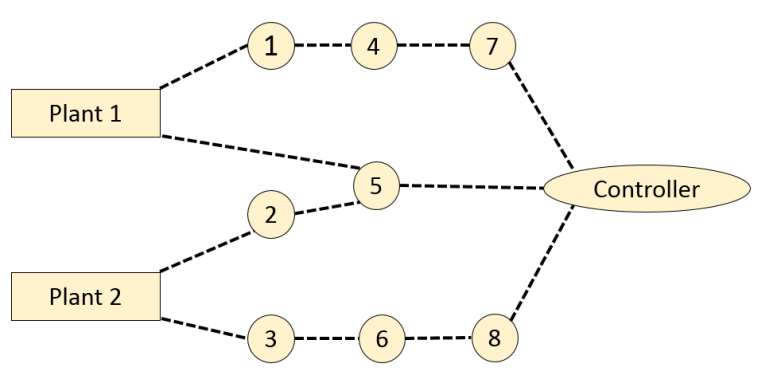

Fig. 4 MHCN considered in a numerical example.

dynamics of Plant 1 and the dynamics of Plant 2 are given by

$$
\begin{aligned}
& x_{1}(k+1)=\left[\begin{array}{cc}
1.08 & 0.1 \\
0.15 & 1.05
\end{array}\right] x_{1}(k)+\left[\begin{array}{l}
0.9 \\
0.5
\end{array}\right] u_{1}(k), \\
& x_{2}(k+1)=\left[\begin{array}{cc}
0.97 & 0 \\
0.3 & 0.99
\end{array}\right] x_{2}(k)+\left[\begin{array}{l}
0.7 \\
0.4
\end{array}\right] u_{2}(k),
\end{aligned}
$$

respectively. Plant 1 is unstable, and Plant 2 is stable.

First, consider modeling an MHCN as a switched linear system. From Fig. 4, $L=4$ holds. Next, the modes are given as follows.

Mode 1: The control input is not sent.

Mode 2: The message about the control input is sent to only Plant 1 through the path (Controller $\rightarrow 5 \rightarrow$ Plant $1)$.

Mode 3: The message about the control input is sent to only Plant 1 through the path (Controller $\rightarrow 7 \rightarrow 4 \rightarrow$ $1 \rightarrow$ Plant 1).

Mode 4: The message about the control input is sent to only Plant 2 through the path (Controller $\rightarrow 5 \rightarrow 2 \rightarrow$ Plant 2).

Mode 5: The message about the control input is sent to only Plant 2 through the path (Controller $\rightarrow 8 \rightarrow 6 \rightarrow$ $3 \rightarrow$ Plant 2).

Mode 6: The path (Controller $\rightarrow 5 \rightarrow$ Plant 1) for Plant 1 and the path (Controller $\rightarrow 8 \rightarrow 6 \rightarrow 3 \rightarrow$ Plant 2) for Plant 2 are chosen.

Mode 7: The path (Controller $\rightarrow 7 \rightarrow 4 \rightarrow 1 \rightarrow$ Plant 1) for Plant 1 and the path (Controller $\rightarrow 5 \rightarrow 2 \rightarrow$ Plant 2) for Plant 2 are chosen.

For each mode, we can obtain a linear system.

Next, setting of the finite-time optimal control problem is explained. The initial control input for both plants is given by 0 . The initial state is given by $x_{1}(0)=\left[\begin{array}{ll}10 & 10\end{array}\right]^{\top}$ for Plant 1 and $x_{2}(0)=\left[\begin{array}{ll}35 & 35\end{array}\right]^{\top}$ for Plant 2. The prediction horizon $N$ is given by $N=2$. The weighting matrices $Q$ and $R$ are given by identity matrices with appropriate sizes.

We present the computational results. Figure 5 and Fig. 6 show the state trajectories and the control inputs, respectively. The mode sequence is obtained as $7 \rightarrow 6 \rightarrow 6 \rightarrow$ $7 \rightarrow 6 \rightarrow 4 \rightarrow 7$. From Fig. 5, we see that the states for both plants converge to the origin.

Circles in Fig. 6 imply the control inputs computed at

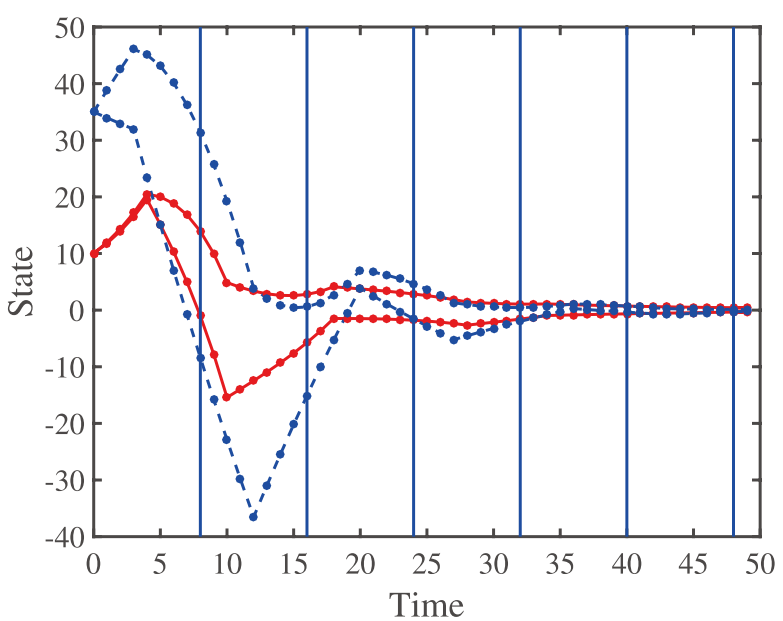

Fig. 5 State trajectories. Solid line: the state of Plant 1. Dash line: the state of Plant 2.

this time. For example, noting that the initial mode is obtained as 7 , the control inputs computed at time 0 are applied to Plant 1 at time 4 and Plant 2 time 3, respectively. In a similar way, noting that the initial mode is obtained as 6 , the control inputs computed at time $8(=2 L)$ are applied to Plant 1 at time 10 and Plant 2 time 12, respectively. Plant 2 is stable, but the initial state of Plant 2 is larger than that of Plant 1. From this fact, the initial mode is chosen as 7 , and the control input for Plant 2 is sent preferentially. At time 8 and time 16, the difference in the state between Plant 1 and Plant 2 becomes small relatively. In this case, since Plant 1 is unstable, the control input for Plant 1 is sent preferentially. Thus, both the control input and the mode can be obtained depending on the current state and the dynamics for each mode.

Finally, we explain the computation time for solving Problem 2 (i.e., the MIQP problem). In this example, Problem 2 was solved seven times. Then, the mean computation time and the worst computation time are $1.51 \mathrm{sec}$ and 2.50 sec, respectively, where we used IBM ILOG CPLEX Optimizer 12.6.2 as an MILP solver on the computer with Intel Core $\mathrm{i} 7-4770 \mathrm{~K} 3.50 \mathrm{GHz}$ processor and $32 \mathrm{~GB}$ memory. Since the candidates of paths are enumerated off-line, Problem 2 can be solved fast. Developing a faster computation method for more complex MHCNs is future work.

\section{Conclusion}

In this paper, a new method of model predictive control (MPC) for a multi-hop control network (MHCN) was proposed. In the proposed MPC algorithm, the timing that both the control input and the path from the controller to plants are recomputed is specified, and these are periodically optimized. Furthermore, a modeling method of switched linear systems that are compatible with the proposed MPC algorithm was proposed. That is, the candidates of paths are enumerated off-line, and for each candidate, a linear system is derived. The effectiveness of the proposed method was 


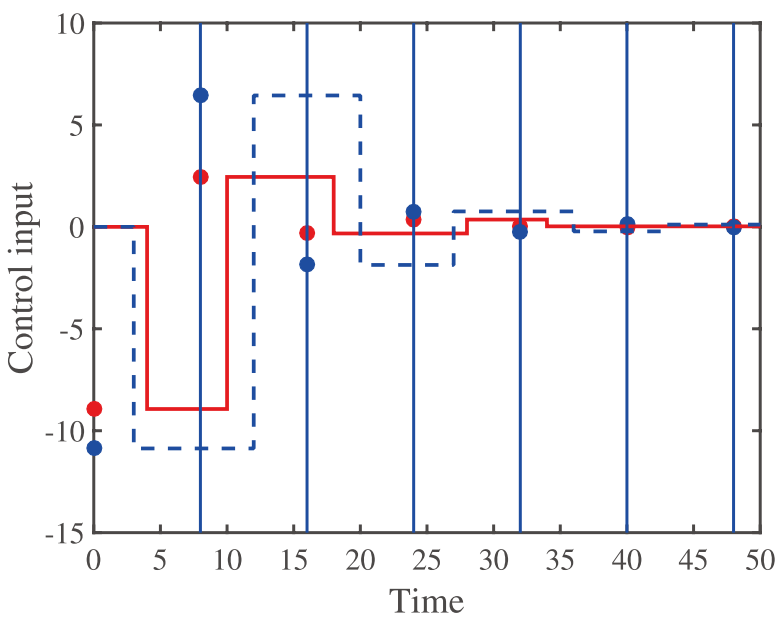

Fig. 6 Control inputs. Solid line: the control input of Plant 1. Dash line: the control input of Plant 2. Circles imply the control inputs computed at $2 L l, l=0,1,2, \ldots$ After the message about the control input reaches the plant, computed control inputs are applied to the plant.

shown by a numerical example.

One of the future efforts is to consider an asynchronous MPC algorithm for MHCNs. In the proposed method, messages about the control inputs for all plants are sent synchronously. This is a strong assumption, and should be relaxed. In asynchronous MPC, the length of cycles may be changed depending on the state of each plant. Hence, centralized management of information about the state is necessary. Multiple controllers interconnected by multi-hop wireless networks can also be utilized. Since the computation time of the finite-time optimal control problem will be long in asynchronous MPC, it is important to develop a distributed optimization algorithm. It is also important to consider model uncertainty and disturbances. Finally, it is significant to consider applying the proposed method to practical applications. The proposed method will be applied to persistent surveillance by multiple agents. Persistent surveillance is that multiple agents continuously and repetitively patrol an area [2]. In particular, the proposed method will be effective for indoor surveillance.

This work was partly supported by JSPS KAKENHI Grant Numbers 26420412, 16H04380.

\section{References}

[1] N. Ahmed, J. Cortes, and S. Martínez, "Distributed control and estimation of robotic vehicle networks," IEEE Control Syst. Mag., vol.36, no.2, pp.36-40, 2016

[2] D. Aksaray, K. Leahy, and C. Belta, "Distributed multi-agent persistent surveillance under temporal logic constraints," Proc. 5th IFAC Workshop on Distributed Estimation and Control in Networked Systems, vol.48, no.22, pp.174-179, 2015.

[3] I.F. Akyildiz, X. Wang, and W. Wang, "Wireless mesh networks: A survey,” Computer Networks, vol.47, no.4, pp.445-487, 2005.

[4] R. Alur, A. D’Innocenzo, K.H. Johansson, G.J. Pappas, and G. Weiss, "Compositional modeling and analysis of multi-hop control networks," IEEE Trans. Autom. Control, vol.56, no.10, pp.23452357, 2011.

[5] P.J. Antsaklis and J. Baillieul, "Special issue on technology of net- worked control systems," Proc. IEEE, vol.95, no.1, pp.5-8, 2007.

[6] A. Bemporad and M. Morari, "Control of systems integrating logic, dynamics, and constraints," Automatica, vol.35, no.3, pp.407-427, 1999

[7] M.D.D. Benedetto, A. D'Innocenzo, and E. Serra, "Fault tolerant stabilizability of multi-hop control networks," Proc. 18th IFAC World Congress, vol.44, no.1, pp.5651-5656, 2011.

[8] T. Braun, A. Kassler, M. Kihl, V. Rakocevic, V. Siris, and G. Heijenk, "Multihop wireless networks," Y. Koucheryavy et al. (eds.), Traffic and QoS Management in Wireless Multimedia Networks, Lecture Notes in Electrical Engineering, vol.31, pp.201-265, 2009.

[9] E.F. Camacho and C.B. Alba, Model Predictive Control, Springer, 2008.

[10] A. D’Innocenzo, M.D. Di Benedetto, and E. Serra, "Fault tolerant control of multi-hop control networks," IEEE Trans. Autom. Control, vol.58, no.6, pp.1377-1389, 2013.

[11] A. Faragó, "Network topology models for multihop wireless networks," ISRN Communications and Networking, vol.2012, Article ID 362603, 23 pages, 2012.

[12] S. Han, Z. Zhong, H. Li, G. Chen, E. Chan, and A.K. Mok, "Codingaware multi-path routing in multi-hop wireless networks," Proc. IEEE Int'l Performance Computing and Communications Conf., pp.93-100, 2008.

[13] K.H. Johansson, G.J. Pappas, P. Tabuada, and C.J. Tomlin, "Guest editorial special issue on control of cyber-physical systems," IEEE Trans. Autom. Control, vol.59, no.12, pp.3120-3121, 2014.

[14] K. Kobayashi and K. Hiraishi, "Optimal control of multi-hop control networks based on the MLD framework," IEEJ Trans. Elec. Electron. Eng., vol.10, no.6, pp.699-705, 2015.

[15] Y. Nanamori and T. Ushio, "Co-scheduling of communication and control of multi-hop control networks," IEICE Trans. Fundamentals, vol.E96-A, no.5, pp.878-885, 2013.

[16] H. Okada, "Multi-route coding in wireless multi-hop networks," IEICE Trans. Commun., vol.E89-B, no.5, pp.1620-1626, 2006.

[17] H. Okada, T. Wada, K. Ohuchi, M. Saito, T. Yamazato, and M. Katayama, "Throughput evaluation of ARQ scheme for multi-route coding in wireless multi-hop networks," Proc. 2006 IEEE 63rd Vehicular Technology Conf., pp.668-672, 2006.

[18] B. Radunovic and J.Y.L. Boudec, "Rate performance objectives of multihop wireless networks," IEEE Trans. Mobile Comput., vol.3, no.4, pp.334-349, 2004.

[19] I. Saha, L.K. Sambasivan, S.K. Ghosh, and R.K. Patro, "Distributed fault-tolerant topology control in wireless multi-hop networks," Wirel. Netw., vol.16, no.6, pp.1511-1524, 2010.

[20] F. Smarra, A. D'Innocenzo, and M.D.D. Benedetto, “Optimal codesign of control, scheduling and routing in multi-hop control networks," Proc. 51st IEEE Conf. Decision and Control, pp.1960-1965, 2012.

[21] E. Witrant, P.G. Park, M. Johansson, C. Fischione, and K.H. Johansson, "Predictive control over wireless multi-hop networks," Proc. 16th IEEE Int'1 Conf. Control Applications, Part of IEEE Multi-conf. Systems and Control, pp.1037-1042, 2007. 


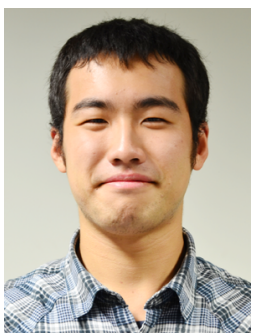

Dai Satoh received the B.E. degree in 2016 from Hokkaido University. Since 2016, he has been pursuing the M.E. degree with the Graduate School of Information Science and Technology, Hokkaido University. His research interests include networked control systems.

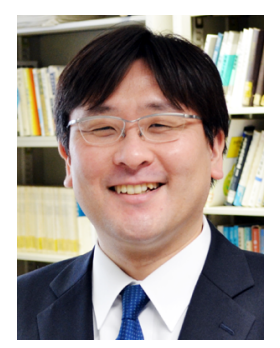

Koichi Kobayashi received the B.E. degree in 1998 and the M.E. degree in 2000 from Hosei University, and the D.E. degree in 2007 from Tokyo Institute of Technology. From 2000 to 2004, he worked at Nippon Steel Corporation. From 2007 to 2015, he was an Assistant Professor at Japan Advanced Institute of Science and Technology. Since 2015, he has been an Associate Professor at the Graduate School of Information Science and Technology, Hokkaido University. His research interests include analysis and control of discrete event and hybrid systems. He is a member of the SICE, ISCIE, IEEJ, and IEEE.

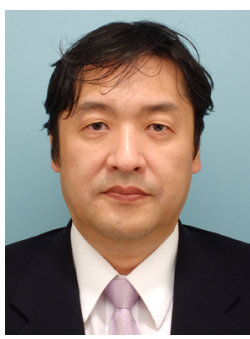

Yuh Yamashita received his B.S., M.S., and $\mathrm{Ph} . \mathrm{D}$. degrees from Hokkaido University, Japan, in 1984, 1986, and 1993, respectively. In 1988, he joined the faculty of Hokkaido University. From 1996 to 2004, he was an Associate Professor at the Nara Institute of Science and Technology, Japan. Since 2004, he has been a Professor of the Graduate School of Information Science and Technology, Hokkaido University. His research interests include nonlinear control and nonlinear dynamical systems. He is a member of SICE, ISCIE, SCEJ, and IEEE. 